\title{
UPPER-AIR FLUORESCENCE AS A TOOL IN \\ X-RAY ASTRONOMY AND SEARCHES FOR X-RAYS FROM NP 0532 AND OTHER PULSARS
}

\author{
W. N. CHARMAN, R. W. P. DREVER, J. H. FRUIN and J. V. JELLEY \\ Nuclear Physics Division, Atomic Energy Research Establishment, Harwell, Berkshire, England \\ and
}

\author{
J. L. ELLIOT, G. G. FAZIO, D. R. HEARN, H. F. HELMKEN, \\ G. H. RIEKE, and T. C. WEEKES
}

Smithsonian Astrophysical Observatory, and Harvard College Observatory, Cambridge, Mass., U.S.A.

\section{Historical Introduction}

The object of this paper, which describes work which has been carried out in close collaboration between members of our two groups, is to present the background of the upper-air fluorescence technique as a viable ground-based tool for studies of cosmic X-rays. After a discussion of the characteristics, merits and limitations of the technique, we describe some preliminary experiments which have been carried out, both separately and also jointly, with our respective installations. These are sited at Mount Hopkins (the SAO field station at $2300 \mathrm{~m}$ altitude near Amado, Arizona) and at Sparsholt Firs (approx. $300 \mathrm{~m}$ altitude, on the Berkshire Downs, near Harwell).

It has long been known that the upper atmosphere fluoresces in the UV, visible and infrared spectral regions under excitation by $\mathrm{X}$-rays. This phenomenon has already been applied effectively to the detection of atomic bombs in space [1,2], through their copious yield of X-rays at the instant of detonation.

The technique is being extensively developed by Greisen and his colleagues at Cornell $[3,4,5]$ as the basis of a method of detecting very large cosmic-ray air showers. In this application, however, the fluorescence is observed at much lower altitudes, altitudes to which primary X-rays could not reach, and at which the fluorescent conversion efficiency is very low, $\sim 10^{-5}$. It is this very extensive work by Greisen which has stimulated interest in this general field, for other applications of the technique to astrophysics.

It was suggested by Colgate [6] that the shock ejection of the outer layers of a supernova should be accompanied by the emission of X-rays and $\gamma$-rays, to a total yield of $\sim 5.10^{47}$ ergs at the source, with an energy spectrum extending to $\sim 2 \mathrm{GeV}$. On the basis of this prediction and estimates of the rate of formation of supernovae in galaxies, Fichtel and Ögelman [7] showed that with relatively simple equipment, it should be possible to detect fluorescent flashes in the upper atmosphere at a sensitivity level yielding a rate perhaps as high as one flash per 8-hour night.

It was with this background of information, and the very thorough laboratory studies of air fluorescence by Hartman [8] that two of the authors of this paper have 
suggested [9] that the technique might well be applied to the search for X-rays from pulsars. Delegates of the Meeting should realise that this work started about Sept. (1968) prior to the discovery by Friedman [10] of the pulsed component of the X-rays from NP 0532, and its subsequent confirmation by many other groups, as reported at this Meeting. While our interest has until now been concentrated predominantly on the pulsars, we are now becoming increasingly enthused by the possibilities of the supernova experiment.

To conclude this section, may we mention that Sir Bernard Lovell has in private correspondence shown considerable interest in the possibilities of detecting X-rays from Flare Stars, already under extensive study in the radio and optical bands of the spectrum.

At this meeting the emphasis is on the search for X-rays from pulsars.

\section{The Atmospheric Fluorescence and its Basic Sensitivity}

The basic idea of the technique is to detect fluorescence in the upper atmosphere generated by cosmic X-rays, the observations to be made with simple optical equipment sited on the ground.

This fluorescence, which occurs predominantly in three wavelength bands, is associated with molecular nitrogen. These bands are the following (i) the 1 st $P$-system of $\mathrm{N}_{2}$, in the infrared, (ii) the 2 nd $P$-system of $\mathrm{N}_{2}$, in the $\mathrm{UV}$, and (iii) the 1 st $N$-system, of $\mathrm{N}_{2}^{+}$, in the visible. Of these, for a variety of reasons, we have only considered (iii).

The bulk of the emission in this, the optical band, occurs within $\Delta \lambda \sim 20 \AA$, at $\bar{\lambda}=3914 \AA$, with a conversion efficiency $\eta=3.4 \times 10^{-3}$ [8] at an altitude $h \sim 65 \mathrm{~km}$. The yield of the isotropically emitted light is approximately linear with the X-ray energy deposited. It has been shown [9] that if the collecting area of the light receiver is $A$ and the solid angle of the field of view is $\Omega$, the signal-to-noise of the system varies as $(A \Omega)^{1 / 2}$, and that with a phototube of given $A$, the optimum performance is attained if this bare phototube is allowed to view the entire upper hemisphere of sky. For a variety of reasons however, certain advantages are in practice gained by the use of an aptical system, consisting for example of a large searchlight mirror with a numerical aperture of typically $f / 0.5$.

The mean lifetime of the electrons, between their photoelectric production by the primary X-rays at say $80 \mathrm{~km}$, to their ionization of the $\mathrm{N}_{2}$ molecules is $\sim 1 \mu \mathrm{sec}$, and the lifetime of the excited $\mathrm{N}_{2}^{+}$ion for emission of the light in the $3914 \AA$ transition is $\sim 100$ n.sec. A further time-dispersion, $\sim$ tens of $\mu \mathrm{sec}$, may occur for an oblique X-ray front to traverse the atmospheric layers encompassed by the field of view of the instrument.

The input energy bandwidth of this type of detector is about three decades, from $\sim 100 \mathrm{eV}$ to $\sim 100 \mathrm{keV}$. The lower limit is set by the steep reduction in the excitation cross-section below $100 \mathrm{eV}$, and the upper limit by collisional de-excitation competing with radiation at the higher pressures, for $E_{x}>100 \mathrm{keV}$. The overall bandwidth may 
be further reduced by interstellar and intergalactic absorption, as discussed elsewhere at this Meeting.

The overall sensitivity of an installation must depend on the various contributions to the total background light from the night-sky, and on the electronic bandwidth; the light pulses have to be observed against the fluctuation noise, and the bandwidth required clearly depends on the duration of the X-ray flashes, and hence on the particular application.

Under an ideal sky, for which the night-sky background light flux is $\phi_{\mathrm{b}}$, and for which the atmospheric transmission is $T$, the shot-noise from the photocathode, of quantum efficiency $\varepsilon_{\mathrm{k}}$ may be calculated directly from the mean cathode current $i_{\mathrm{k}}$ expressed in photoelectron emission rate $\left(\mathrm{d} N_{\mathrm{e}} / \mathrm{d} t\right)$, so that

$$
i_{\mathrm{k}}=\left(\mathrm{d} N_{\mathrm{e}} / \mathrm{d} t\right)=A \Omega \int \phi_{\mathrm{b}}(\lambda) T(\lambda) \varepsilon_{\mathrm{k}}(\lambda) \mathrm{d} \lambda
$$

and the mean square value of the shot noise is:

$$
<\Delta i_{\mathrm{k}}^{2}>=2 e i_{\mathrm{k}} \cdot \delta f
$$

in a bandwidth $\delta f$.

To the shot noise must be added other contributions to fluctuations in the light of the night-sky, many of which have already been discussed by us elsewhere [9].

Since most of the fluorescence occurs in a narrow band, the sensitivity can be raised by the use of narrow band filters, though in general interference filters are excluded, by the large values of $\Omega$. Gelatine or glass filters will nevertheless enhance the sensitivity. For example, the combination of an S-11 cathode and a Corning 'Amethyst' filter, type 7-62, provides an admirable combination.

\section{Merits and Limitations}

It is essential to realise that the technique is limited to X-ray sources for which there are variable components of the flux which occur on time-scales short compared with those associated with fluctuations in the sky background. The applications are therefore restricted to supernovae, pulsars, variable X-ray sources and flare stars. The time-scales involved are approximately as follows:

Supernovae, $\sim$ tens of $\mu$ secs [6]

Pulsars, frequency components $\sim 10 \mathrm{~Hz}$ to $5 \mathrm{kHz}$

Variable X-ray sources (e.g. Sco X-1) mins

Flare stars, $\sim$ minutes to hours.

In general the lower the frequencies the greater the problems.

The chief merits of the fluorescence technique are:

(1) Large input energy bandwidth, $\sim 1 \mathrm{keV}-100 \mathrm{keV}$, with an additional decade, $100 \mathrm{eV}-1 \mathrm{keV}$, limited by interstellar and intergalactic absorption [11].

(2) Long integrating times, $\sim 6 \mathrm{hr}$ per night, for unlimited periods.

(3) Ground-based, simple, low cost, and

(4) Since the fluorescent light is emitted isotropically, the detectors individually 
are non-directional, so that all sources present above the horizon may be observed simultaneously. In the case of the supernova application, widely spaced stations can however be used to obtain directional information to locate galaxies to a precision $\sim 1^{\circ}$, a resolution small compared with the dimensions $\left(\sim 10^{\circ} \times 10^{\circ}\right)$ of the larger clusters of galaxies [12].

The disadvantages of the technique are:

(1) A single detector is non-directional.

(2) The performance is sensitive to site, climate and local weather.

(3) The system is difficult to calibrate.

(4) There is no spectral information.

With these considerations in mind, each application has its own particular problems which are summarised briefly as follows:

Supernovae. Technically straightforward, but the expected relatively low event-rate demands the organisation of a network of widely spaced stations, to ensure avoidance of local optical interference (lightning, meteors and man-made flashes) and to obtain an adequate coincidence probability of clear skies at two or more sites.

Pulsars. By far the greatest problem is that the first harmonic of the line-frequencies, 100 or $120 \mathrm{~Hz}$, lies in precisely the electronic bandwidth required, so that it is essential to site the installations far from sources of A.C. lighting.

Variable $X$-ray sources and flare stars. Since the time-scales for these phenomena are very long, it is anticipated that the sensitivity will be limited by the constancy of the mean level of the sky-background over the periods required.

\section{Preliminary Experiments at Harwell and Mt. Hopkins}

We will now describe briefly some preliminary experiments which have been carried out at Harwell, starting in September of 1968, and on Mt. Hopkins, around November, 1968.

\section{A. THE HARWELL EXPERIMENT}

A $150-\mathrm{cm}$ dia. $f / 0.5$ parabolic searchlight reflector was used with a $30-\mathrm{cm}$ dia. EMI phototube (type 9545B) having an S-11 cathode of sensitivity $\sim 50 \mu \mathrm{A} /$ lumen; no filter was used. The analogue signals from the tube were amplified, filtered $(\Delta f \sim 2 k \mathrm{~Hz})$, digitised and recorded on magnetic tape, along with a clock reference signal derived from an ovened $5 \mathrm{MHz}$ crystal, stable to 1 in $10^{8}$ and calibrated against the $200 \mathrm{kHz}$ carrier of the B.B.C. Droitwich transmitter. The tapes were read out into a 512channel Laben analyser cycled from the clock reference via a vernier scaler system [13]. The tapes were read out several times, for periods corresponding to those for the various pulsars, these periods being corrected for orbital and axial rotation Doppler shifts. The installation was sited at Sparsholt Firs, a dark site several kilometers from nearby villages and towns. In spite of this, residual $100 \mathrm{~Hz}$ 'hum' was considerable, though this was eventually quenced by hum-bucking, as shown in Figure 1, which illustrates the essential features of the recording system. 
Runs were carried out on a number of occasions, under a variety of conditions and selection of the various parameters, and the tapes were analysed for the periods of the pulsars NP 0532, CP 1133, and CP 0950.

The best overall conditions occurred on Jan. 18-19 (1969) when a run was obtained

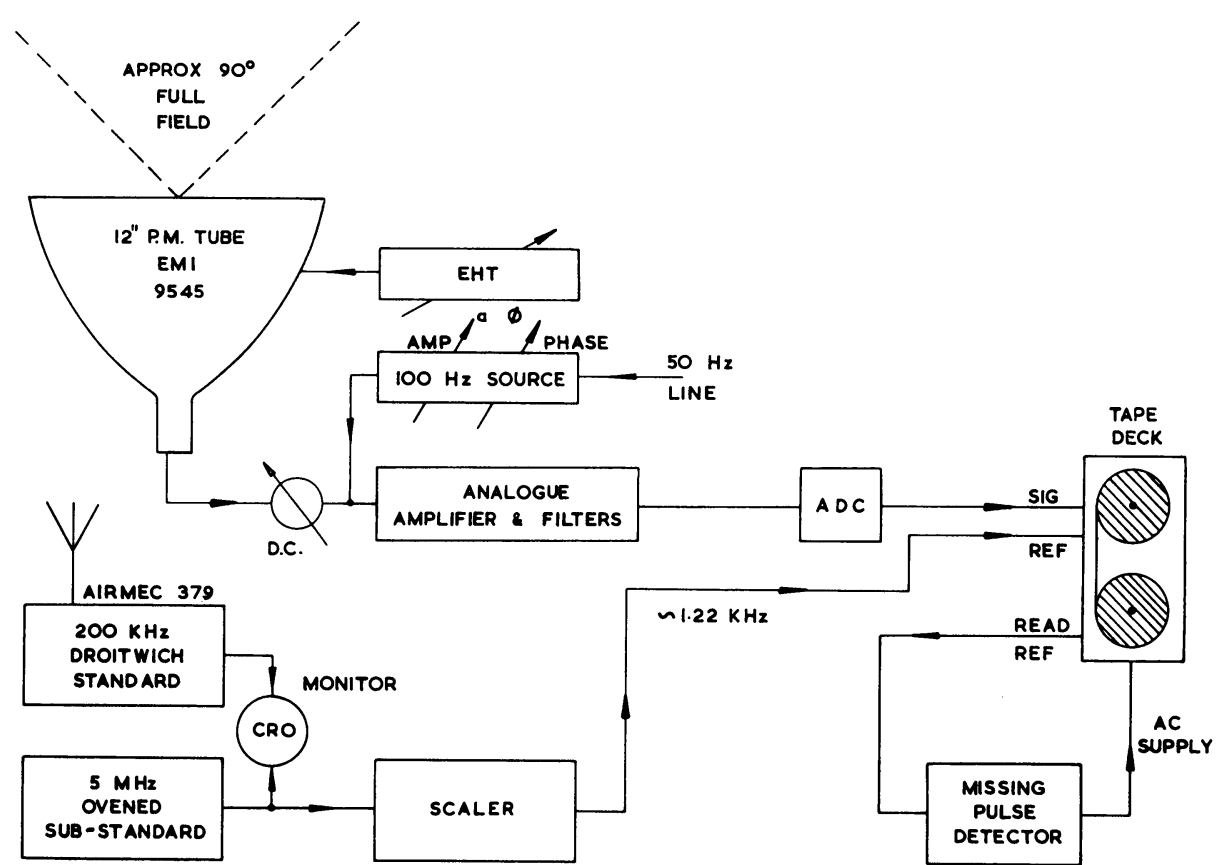

Fig. 1. The basic features of the recording system used at AERE for a search for X-ray fluorescent pulses from the three pulsars. As used during the runs of Jan. 18-19th, a 120-cm diam. searchlight mirror was used, and $\mathrm{a} \div 10^{3}$ scaler was interposed between the ADC and the recorder.

for approximately three hours, broken into three periods for which the phase was preserved during each of these periods.

So far, none of these observations have as yet on analysis yielded any evidence for pulses at a significant level, and it is hoped the observations will be continued next winter, particularly as there is now firm evidence for X-ray pulses from NP 0532 at a variety of energies.

During these observations low frequency components were frequently seen. These, discussed by the SAO group, may have contributed additional noise to the basic shot-noise, thus lowering the sensitivity.

Theoretical sensitivity for NP 0532

For the above installation, $A \Omega=2200 \mathrm{~cm}^{2} \mathrm{sr}$. For the $S-11$ cathode $\bar{\lambda} \sim 4000 \AA$, $\delta \lambda \sim 1500 \AA$ ( $3 \mathrm{db}$ points $)$, and $\bar{\varepsilon}_{\mathrm{k}} \sim 0.1$. In this bandwidth, $\int \phi \mathrm{d} \lambda=6.4 \times 10^{7}$ photons $\mathrm{cm}^{-2} \mathrm{sec}^{-1} \mathrm{sr}^{-1}$ [14]. With $T_{4000}=0.63$ [15], and the above figures, we find from (1) 
that

$$
\left(\mathrm{d} N_{\mathrm{e}} / \mathrm{d} t\right)=8.8 \times 10^{9} \text { photoelectrons } \mathrm{sec}^{-1} .
$$

Let us consider NP 0532, for which the main pulse has width $\Delta t \sim 2 \mathrm{msec}$, and $P \sim 33 \mathrm{msec}$. We therefore require the analyser to have say 20 channels. Consider now an integration period of $t=1 \mathrm{hr}$. In this time we accumulate $3.2 \times 10^{13}$ counts or $1.6 \times 10^{12}$ counts per bin, with $\sigma= \pm 1.3 \times 10^{6}$.

For a $1 \%$ confidence level over 20 channels, we require that one of these channels has a fluctuation $\geqslant 3.5 \sigma$ or $\geqslant 4.6 \times 10^{6}$ photoelectrons or $\geqslant 4.6 \times 10^{7}$ photons in an hour's run. Since $1 h v$. at $3914 \AA \equiv 3.2 \mathrm{eV}$, this represents a time-averaged light flux in the pulses, of

$$
\phi_{\mathrm{L}} \geqslant 18.6 \mathrm{eV} \mathrm{cm}^{-2} \mathrm{sec}^{-1} \mathrm{sr}^{-1} \text {. }
$$

With a conversion efficiency $\eta=3.4 \times 10^{-3}$ and assuming the light may be emitted isotropically over $4 \pi$, this represents a minimum detectable X-ray flux, over any part of the band approx. $1 \mathrm{keV}-100 \mathrm{keV}$, of

$$
\Phi_{\mathrm{x}}=6.9 \times 10^{4} \mathrm{eV} \mathrm{cm}^{-2} \mathrm{sec}^{-1}
$$

or a differential sensitivity of

$$
\phi_{\mathrm{x}} \geqslant 4.6 \times 10^{-27} \mathrm{erg} \mathrm{cm}^{-2} \mathrm{sec}^{-1} \mathrm{~Hz}^{-1} \text {. }
$$

The light yield from a complete spectrum of form $\phi_{\mathrm{x}}=k E^{-\gamma} \cdot \mathrm{d} E$ is then

$$
\Phi_{\mathrm{L}}=k \int_{E_{1}}^{E_{2}} \cdot \eta(E) \cdot E^{-\gamma} \cdot \mathrm{d} E .
$$

In Figure 2 are plotted the spectral points obtained by rocket and balloon flights which have been presented at this Meeting, along with others already published. A reasonably good fit is obtained in the energy-range of interest, by taking a power-law with $\gamma=1.0$ and between $E=1 \mathrm{keV}$ and $E=100 \mathrm{keV}$. If we now take our limiting sensitivity (3) and a spectrum of the same slope as that of the observed spectrum, we obtain the line $b b^{\prime}$ in Figure 2, showing that the overall sensitivity lies somewhat outside the errors of existing measurements on NP 0532, for an hour's integration. The addition of filters and an increased integration time should bring the source within detectable range, expecially as some relaxation on the statistics is permitted, when allowance is made for the known double-pulse structure of the expected signal, with the characteristic $14 \mathrm{msec}$ spacing.

That no positive effects have been seen on the experimental runs so far, is attributed to a number of limitations, among which were (i) loss of performance due to residual hum, (ii) degradation of statistics in pre-scaling between the ADC and the recorder, and (iii) non-Gaussian noise at the lower frequencies, from the night-sky, as mentioned below.

\section{B. THE SMITHSONIAN ASTROPHYSICAL OBSERVATORY (SAO) EXPERIMENT}

Four $130 \mathrm{~mm}$ diameter phototubes, two of type RCA 4522 and two of type Amperex 


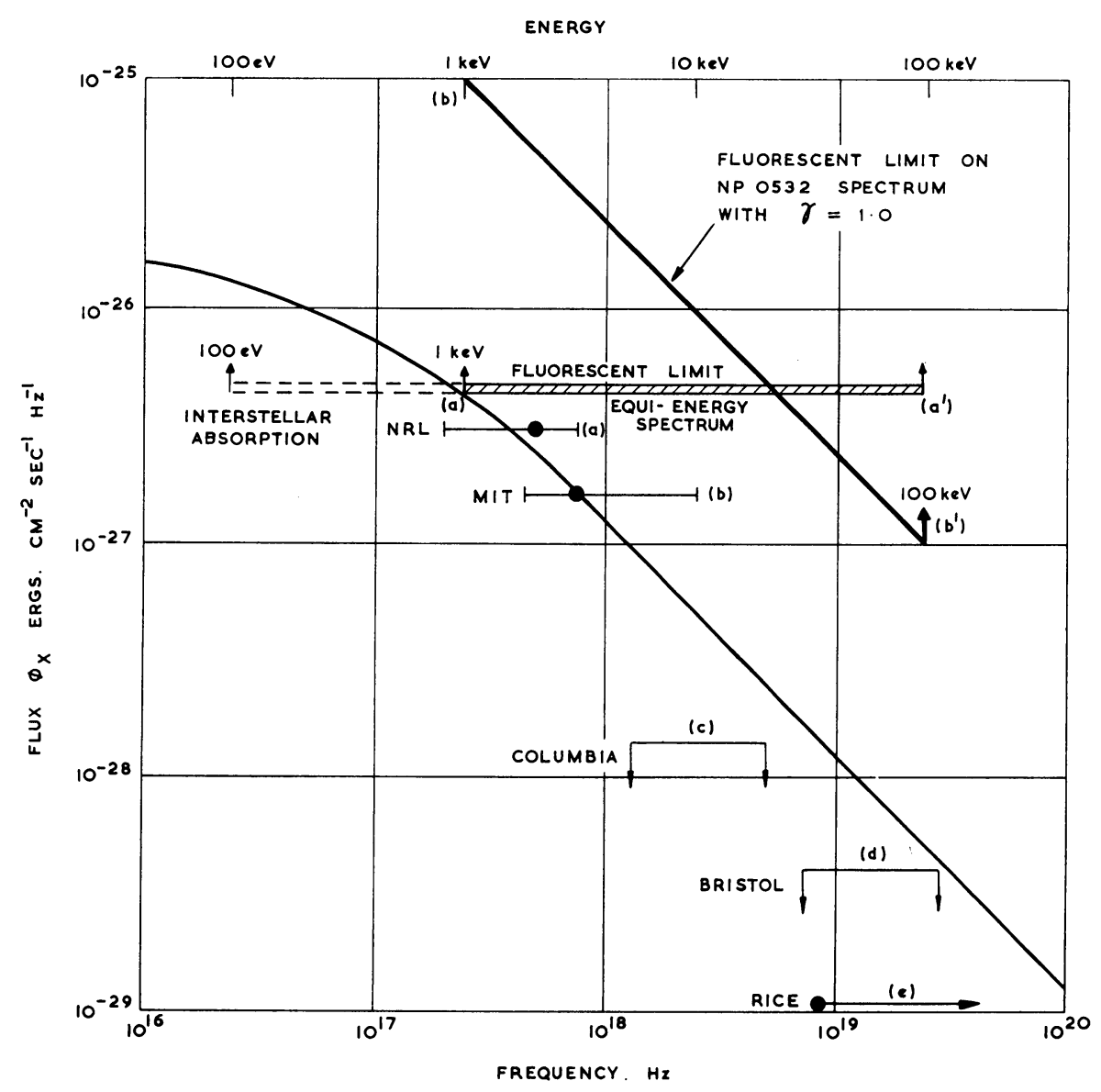

Fig. 2. The differential spectrum of X-rays from the pulsar NP 0532. (a) The spectral point obtained by N.R.L. and reported by H. Friedman at this Meeting (this volume, p. 34, also ref. [16]). (b) The results of the MIT experiment, as reported by B. Rossi at this Meeting (this volume, p. 1, also ref. [17]). (c) The Columbia results, from the work reported by R. Novick at this Meeting (this volume, p. 145). This upper limit flux, of $\leqslant 5 \%$ of the general Crab background, refers however only to the component of the pulsar radiation at the fundamental frequency of $30 \mathrm{~Hz}$. (d) The upper limit flux reported by the Bristol group, ref. [18]. (e) This result, from the group at Rice, was reported at this Symposium by R. C. Haymes (this volume, p. 185, also ref. [19]). $a a^{\prime}$ represents the theoretical limiting sensitivity calculated by the authors of this paper, for a 1 hour's integration of the fluorescence light of the upper atmosphere, in a $1 \mathrm{kHz}$ bandwidth, with a $150 \mathrm{~cm}$ diam. $f / 0.5$ searchlight and a $30-\mathrm{cm}$ diam. EMI phototube of quantum efficiency $\sim 10 \%$. The curve $b b^{\prime}$ represents the sensitivity of the same instrument when exposed to a spectrum having the same slope $(\gamma \sim 1.0)$ as that observed in the rocket and satellite experiments reported at this Meeting. The curves $a a^{\prime}$ and $b b^{\prime}$ are derived assuming a perfect dark sky for which (i) the power spectrum is flat throughout the frequency band considered i.e. $\sim 10 \mathrm{~Hz}-3 \mathrm{kHz}$, and (ii) that the sky is totally free from line-frequency hum. The optical point on the spectrum, off the graph itself, is derived from the work of Lynds et al. [20] with a time-averaged flux of $\bar{m}_{\mathrm{v}}=16.5, B-V=0.6$ and $m_{B}=17.1$, using the absolute flux at $m_{B}=0$ of $\phi_{\text {opt }}=6.5 \times 10^{-9} \mathrm{erg} \mathrm{cm}^{-2} \mathrm{sec}^{-1} \AA^{-1}$ at $\lambda=4401 \AA$ obtained by Willstrop (Monthly Notices Roy. Astron. Soc. (1960) 121, 17). The spectral point, after correction of $1.1 \mathrm{mag} \mathrm{kpc}^{-1}$ for absorption, is at a flux level of $\phi_{\text {opt }}=3.7 \times 10^{-26} \mathrm{erg} \mathrm{cm}^{-2} \mathrm{sec}^{-1} \mathrm{~Hz}^{-1}$ at $v_{\text {opt }}=6.85 \times 10^{14} \mathrm{~Hz}$. 
58 AVP were mounted in a light screen to view the zenith-sky, so that the total sensitive area $A$ was $402 \mathrm{~cm}^{2}$ and the effective solid angle $\Omega$ was $2.36 \mathrm{sr}$. The analogue signals from the tubes were linearly added, amplified and filtered $(\Delta f \sim 1 \mathrm{kHz})$, then linearly converted to frequency by a voltage controlled oscillator. The output of the oscillator was recorded on one channel of magnetic tape, while $1 \mathrm{kHz}$ clock pulses were recorded on the second channel. The clock signals were obtained from the SAO satellite tracking station at Mt. Hopkins and are stable to one part in $10^{10}$. The magnetic tapes were analyzed on a 100-channel pulse height analyzer operating in the multiscalar mode. The analyzer was recycled every two pulsar periods from the clock pulses via a vernier scaler system [13]. The pulsar period was corrected for earth orbital and axial rotation.

The experiment was located at the $2300 \mathrm{~m}$ elevation of Mt. Hopkins, Arizona. The data were recorded under very dark site conditions and during periods of excellent sky transparency. A $120 \mathrm{~Hz}$ signal recorded due to distant city lights was present, but at a low level.

Data were taken over four nights from November 18 through November 21, 1968, and approximately 33 hours of data were recorded, with each tape reel lasting about 1.5 hours. Absolute time marks were also recorded on each tape but no effort was made to add successive tapes coherently.

The data were analyzed only for the pulsar NP 0532 (Crab Nebula). Thus far these tapes have yielded no evidence of X-ray pulsations, with an upper limit to the X-ray flux of $2.5 \times 10^{4} \mathrm{eV} \mathrm{cm}^{-2} \mathrm{sec}^{-1}$.

A most interesting aspect of the data taken at Mt. Hopkins is the power-spectrum (square of the amplitude of the Fourier components in a given frequency interval) of the night airglow over the frequency range from 0 to $600 \mathrm{~Hz}$. The amplitude of the power spectrum is not constant at all frequencies, as would be expected, but is higher in the 0 to $100 \mathrm{~Hz}$ region, at a level about 3 to $10 \mathrm{db}$ higher than the rather flat spectrum above $200 \mathrm{~Hz}$. Thus, in the band where most of the pulsar frequency components are expected, the night sky light fluctuations appear to be higher than would be expected from purely statistical effects. The sensitivity is not as great, therefore, as theoretically calculated in the previous section. The origin of the excess components below $100 \mathrm{~Hz}$ is completely unknown to us, and must be investigated further if the present upper limits are to be lowered significantly.

\section{Conclusions}

It is concluded that with care it should be possible to detect the X-rays from NP 0532 by ground-based observations of air fluorescence, with integration times $\sim$ hours.

Of the other suggested applications, the search for supernovae by this technique is particularly attractive. The estimates of expected counting rates [7], a few per week, make it a viable experiment, and the simultaneous operation of a number of stations spaced $\sim 1000 \mathrm{~km}$ apart would yield a directional accuracy $\sim 1^{\circ}$, with recording of real time to a precision of $\sim 100 \mu \mathrm{sec}$. This resolution is adequate for astronomical 
search purposes and is considerably less than the angular sizes of the larger clusters of galaxies, $\sim 10^{\circ}$. The recording of real time to $\sim 100 \mu \mathrm{sec}$ is straightforward, using VLF transmissions from stations such as WWV, MSF or HBG.

Since the recordings would be made in a frequency band $\delta f \sim 100 \mathrm{kHz}-1 \mathrm{MHz}$, the technique should not be plagued by the background of line-frequency components of the light of the night-sky, or by other low-frequency fluctuations.

The application to the detection of supernovae has obvious astrophysical interest, as it presents a direct test of Colgate's model of the shock-wave phenomenon, and, if successful, could be developed to alert astronomers for optical observations of the early phases of the subsequent light-curve.

\section{References}

[1] Westervelt, D. R. and Hoerlin, H.: 1965, Proc. IEEE 53, 2067.

[2] Donahue, T. M.: 1965, Proc. IEEE 53, 2072.

[3] Greisen, K.: 1965, in Proc. 9th Internat. Conf. on Cosmic Rays, London, p. 609.

[4] Bunner, A. N.: 1966, Cornell-Sydney University Astronomy Center Report No. 62, also Cornell thesis.

[5] Jenkins, E. B.: 1966, Center for Radiophysics and Space Research, Cornell, N.Y., Report No. CRSR 250, also Cornell thesis.

[6] Colgate, S. A.: 1968, Canad. J. Phys. 46, S476.

[7] Fichtel, C. E. and Ögelman, H. B.: 1968, NASA Technical Note No. 4732.

[8] Hartman, P. L.: 1968, Los Alamos Scientific Lab. Report LA-3793.

[9] Fazio, G. G. and Jelley, J. V.: 1969, AERE Rep. No. 6095.

[10] Friedman, H.: IAU Telegramme No. 2141, also this volume, p. 34.

[11] Felten, J. E. and Gould, R. J.: 1966, Phys. Rev. Lett. 17 (7), 401.

[12] Abell, G. O.: 1965, Ann. Rev. Astron. Astrophys. 3, 1.

[13] Papaliolios, C., Carleton, N. P., Horowitz, P., and Liller, W.: 1968, Science 160, 1104.

[14] Jelley, J. V.: 1967, Prog. Elem. Particle Cosmic Ray Phys. IX, 53.

[15] Allen, C. W.: 1955, Astrophysical Quantities, Univ. of London, Athlone Press, 1st ed., p. 116.

[16] Fritz, G., Henry, R. C., Meekins, J. F., Chubb, T. A., and Friedman, H.: 1969, Science 164, 709.

[17] Bradt, H., Rappaport, S., Mayer, W., Nather, R. E., Warner, B., McFarlane, M., and Kristian, J.: 1969, Nature 222 (5195), 728.

[18] Hillier, R. R., Jackson, W. R., Murray, A., Redfern, R. M., and Standing, K. G.: 1969, Nature 222 (5189), 149.

[19] Fishman, G. J., Harnden, F. R., and Haymes, R. C.: 1969, Astrophys. J., in press.

[20] Lynds, R., Maran, S. P., and Trumbo, D. E.: 1969, Astrophys. J. 155, L. 121. 